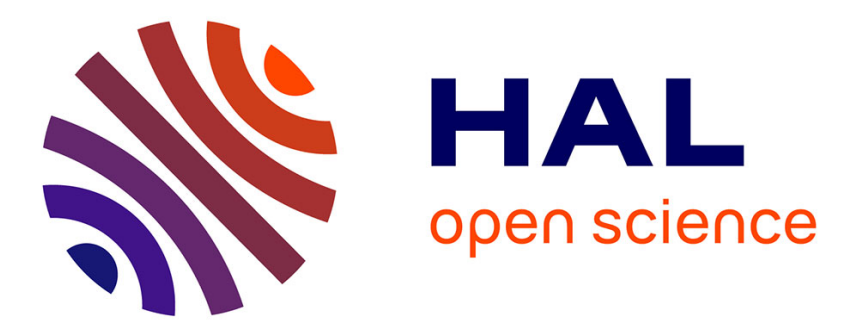

\title{
Epitaxial Magnetic Garnet Heterostructures
}

R. Ramesh, B. Simion, G. Thomas

\section{- To cite this version:}

R. Ramesh, B. Simion, G. Thomas. Epitaxial Magnetic Garnet Heterostructures. Journal de Physique IV Proceedings, 1997, 07 (C1), pp.C1-695-C1-698. 10.1051/jp4:19971284 • jpa-00254997

\section{HAL Id: jpa-00254997 https://hal.science/jpa-00254997}

Submitted on 1 Jan 1997

HAL is a multi-disciplinary open access archive for the deposit and dissemination of scientific research documents, whether they are published or not. The documents may come from teaching and research institutions in France or abroad, or from public or private research centers.
L'archive ouverte pluridisciplinaire HAL, est destinée au dépôt et à la diffusion de documents scientifiques de niveau recherche, publiés ou non, émanant des établissements d'enseignement et de recherche français ou étrangers, des laboratoires publics ou privés. 


\title{
Epitaxial Magnetic Garnet Heterostructures
}

\author{
R. Ramesh, B.M. Simion* and G. Thomas** \\ Department of Materials and Nuclear Engineering and Center for Superconductivity Research, \\ Department of Physics, University of Maryland, College Park, MD 20742, U.S.A. \\ * Seagate Technology, Inc., Bloomington, MN 55435, U.S.A. \\ * Department of Materials Science and Mineral Engineering, University of California, Berkeley, \\ CA 94720-1760, U.S.A.
}

Abstract : A summary of our recent progress in the growth and characterization of magnetic and magnetooptic garnet heterostructures is presented in this paper. The similarity in crystal chemistry and lattice parameters among the various garnets (yttrium iron garnet, bismuth iron garnet, etc.) is utilized to create artificially layered heterostructures and superlattices. Rare earth (especially Eu) substitutions in the $\mathrm{Bi}$ site are shown to dramatically improve the magnetooptic response and change the easy axis from in-plane to out-of-plane. Microstructural studies indicate that the interface roughness is dependent on the thicknesses of the different layers suggesting the possible role of lattice mismatch induced stresses in surface roughening.

\section{INTRODUCTION}

There is now considerable research effort directed towards the growth, characterization and device fabrication of thin film magnetic oxides. These oxides which are the perovskites, garnets, spinel ferrites or hexaferrites, have found use in a vast variety of technological applications[1]. Thick films of magnetic garnets were grown in the sixties and seventies for bubble memory applications[2]. These films were grown typically by liquid phase epitaxy onto lattice matched substrates such as GGG in the thickness range of a few micrometers. More recently, the observation of very large values of magnetooptic Kerr rotation angles in thin films (with thickness $\leq 1 \mu \mathrm{m}$ ) of Bismuth doped Yttrium Iron Garnet (YIG) [3] has fueled research excitement in this area, since it is a key requirement not only for high density, non-volatile magneto-optic (MO) memories, but also for optical isolator and rotator applications. The potential for obtaining very desirable magneto-optic properties in conjunction with their transparency makes them very attractive candidates for multi-layered MO media. A comprehensive understanding of the effect of granularity of the magnetic film, or conversely crystallinity, and defect chemistry on the high frequency and magnetooptic performance of these magnetic oxides is an important aspect and deserves serious attention.

The magnetooptic properties of ferromagnetic oxides form the basis for another technologically important area, namely nonvolatile information storage [3-7]. The relatively large values of Faraday rotation angles obtained in sub-micron thin films has fueled research and development effort in this area. The large values of Faraday rotation coupled with the intrinsic corrosion / oxidation resistance in these materials is an advantage over the rare earth-transition metal alloys. However, there are several materials, processing and microstructure related problems that have to be addressed before commercially viable storage systems, using the garnets as the storage media, can be realized. Among them is the fact that these films have to be processed at high temperatures. This obviates the use of polycarbonate substrates that are now being used for the optical recording media. Since cost is an important factor and hence inexpensive glass substrates are being studied as potential substrates, significant effort needs to be aimed at studying the microstructural evolution and the interrelationship between microstructure-processing and magnetooptic performance. Another more important issue is that related to the noise in the media, which is mainly due to one of two reasons : (i) light scattering from impurities in the grain boundaries in the polycrystalline garnet films; and (ii) irregularity in the thermomagnetically written bit due to low domain wall coercivity inside the grains. The exact role of grain boundaries, chemical impurities at these internal interfaces and the effect of film microstructure on the magnetooptic performance still needs to be understood.

In recent years, there has been an increasing awareness that the magnetic properties of thin films are significantly different from those of the bulk, primarily due to enhanced contributions from surfaces and interfaces $[8,9]$. One of the methods to study the contributions of internal interfaces and surfaces has been to grow multi-layer stacks of magnetic layers interleaved with magnetic or non-magnetic layers. In such superlattices which are comprised of ultrathin magnetic layers, the interface contribution to the overall magnetic anisotropy can be strong enough to induce a perpendicular magnetic anisotropy, although the shape anisotropy dictates an in-plane magnetization. The interrelationship between the film growth mechanism, the layer thicknesses and the nature of the different layers governs the final magnetic properties of the film and needs to be established through systematic experiments. This paper reviews some of our recent progress in the growth of garnet heterostructures bu pulsed laser deposition (PLD), characterization of their microstructural and magnetic/magneto-optic properties.

\section{EXPERIMENTAL DETAILS}

The garnet thin films are grown by in-situ PLD on single crystal [111] GGG substrates. Details of the experimental procedures are already presented elsewhere[10,11]. Fig.1 illustrates schematically the heterostructures that are being grown in this program, and depicts the close similarity in crystal chemistry and lattice parameters that enable heteroepitaxy in this system. 


\section{RESULTS}

We have carried out a considerable amount of structural and microstructural characterization of these thin film heterostructures in order to understand the effect of processing parameters such as layer thicknesses, oxygen pressure and lattice mismatch with the substrate. Diffraction contrast imaging is especially useful in differentiating between the two

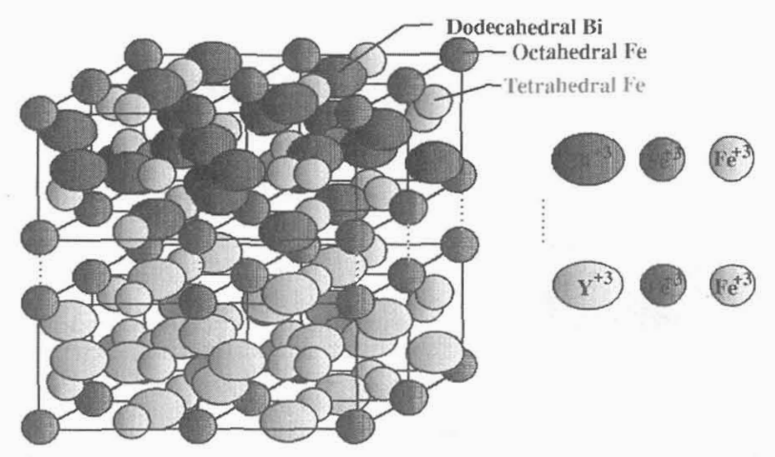

Figure 1: A schematic of the crystal structure of garnets showing the similarity in crystal chemistry and lattice registry leading to heteroepitaxy.

types of layers as illustrated in Fig.2(a), in which the Eu-BIG layer appears in darker contrast compared to the YIG layer. These TEM studies in conjunction with high resolution imaging show the interfaces between the YIG and Eu-BIG layers to be free of reaction products. However, if the thicknesses of the two types of layers is altered, namely if the Eu-BIG layer is made thicker, the heterostructures appear wavy as they thicken, as illustrated in Fig.2(b), suggesting the possibility of strain relaxation through a long range structural modulation. Further work is required to fully understand this effect.
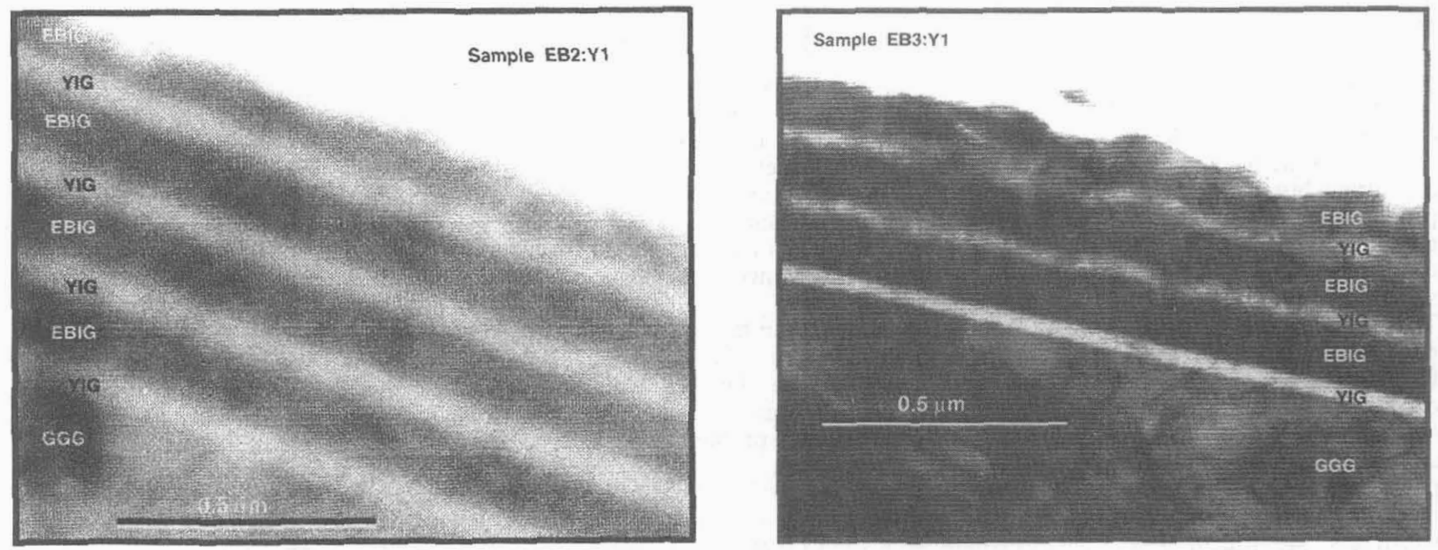

Figure 2 : (a) TEM image of a Eu-BIG/YIG superlattice with a thickness ratio of 2:1 showing smooth interfaces ; (b) TEM image of a Eu-BIG/YIG superlattice with a thickness ratio of $3: 1$ showing the "wavy" surface structure.

Preliminary work in our group on the laser deposition of garnet thin films has shown that the properties are very sensitive to the deposition parameters including the oxygen pressure, the deposition temperature and the nature of the rare earth in the garnet structure. For example, we find that single layer YIG films and YIG/BIG heterostructures generally have a preferred in-plane magnetization. Furthermore, the in-plane loop squareness is very dependent on the oxygen pressure during the deposition. If part of the Yttrium or Bismuth site is substituted with another rare earth such as Eu, we find that the films and heterostructures have a strong preference for out-of -plane magnetization, as illustrated in Fig.3. Furthermore the coercive field also becomes much higher (reaching values higher than 1kOe in some samples) in these films compared to the films without Eu. Further studies using other rare earth substitutions are in progress to enable understand the origin of this change in preferred magnetization direction.

These films also show very desirable magnetooptic characteristics. Fig.4 shows typical magnetooptic hysteresis loops obtained from three samples, as described in the figure. This figure also serves to illustrate that many factors may play a role in determining the nature of the magnetooptic response. An interesting aspect that is related to the growth of the thin film heterostructures is also illustrated in this figure. When a single layer Eu ${ }_{1} \mathrm{Bi}_{2} \mathrm{Fe}_{5} \mathrm{O}_{12}$ (EBIG) film is grown that magnetooptic response is not as large (after thickness normalization) as the film which is grown on a very thin YIGtemplate layer. This approximately $300 \AA$ thick template layer, which is grown directly on the GGG single crystal surface, appears to provide a better chemical environment for the subsequent growth of the EBIG layer. These preliminary 
results clearly show that high quality garnet thin films and superlattice heterostructures can be grown and provide the potential for systematic variations, control and finally engineering of the interlayer interfaces.

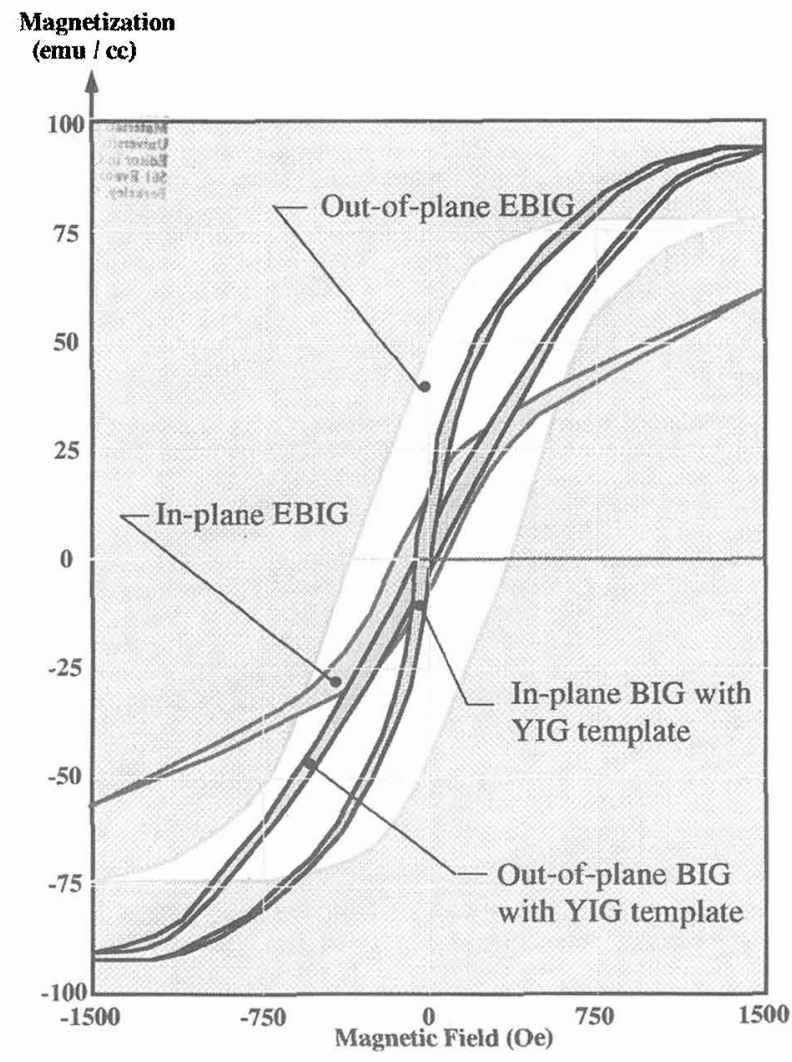

Figure 3: In-plane and out-of-plane magnetization hysteresis loops for the BIG and Eu-BIG thin films grown on YIG substrates. Note that the film with Eu substitution is strongly out-of-plane magnetized. Kerr Rotation

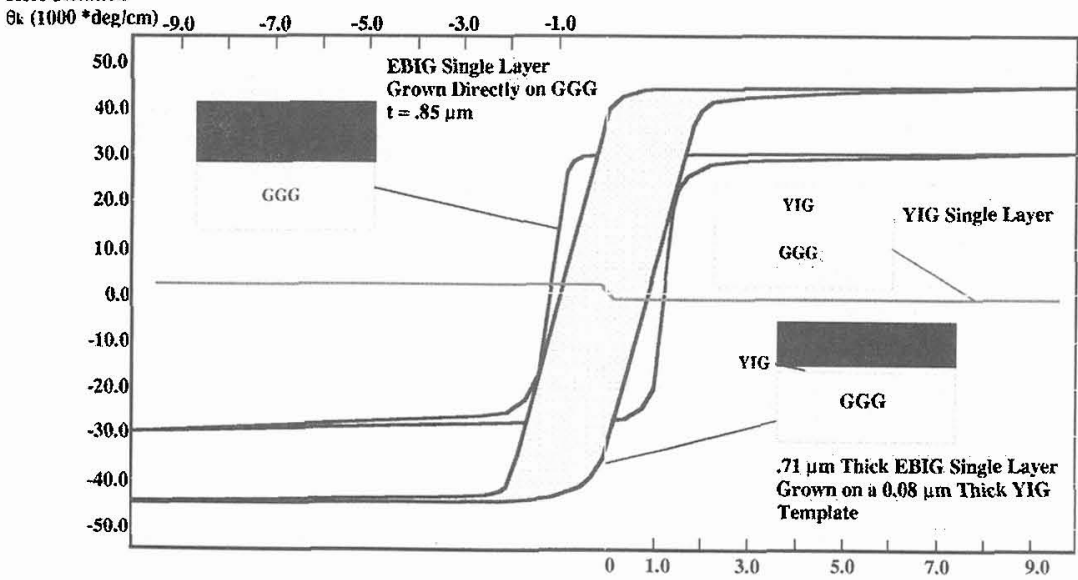

Applied Field (kOe)

Figure 4: Faraday rotation as a function of applied field for the single layer YIG and Eu-BIG films compared with the loop for a Eu-BIG film grown using a thin YIG template layer. 


\section{SUMMARY AND FUTURE DIRECTIONS}

This brief review clearly demonstrates that high quality epitaxial garnet heterostructures can be grown using PLD as the deposition tool. These heterostructures show magnetic and magnetooptic responses that are both scientifically challenging and technologically interesting. Many issues still remain to be understood and resolved. These include the role of the rare earth species in determining the preferred magnetization direction. It is not clear whether this is driven by the magnetocrystalline anisotropy associated with the rare earth ion or due to growth induced stresses. The effect of heteroepitaxial layering in enhancing the magnetic and magnetooptic responses has to be further clarified. The question of the physical state of the interfaces seems to be also related to roughness and magnetic coupling. All these films possibly retain internal stresses and therefore mechanisms of stress release, i.e., twinning, microcracking or surface roughening are likely to be very important. In addition, advanced electron microscopy techniques to understand site occupancies (specifically RE-Bi cationic sites) need to be explored to understand the origins of the magnetic and magnetooptic properties. From the basic thin film growth point of view, the origin of the "wavy" (i.e., the long range structural modulation) due to changes in the Eu-BIG layer thickness needs to be understood. Finally, the reasons for the beneficial effects of the YIG "template" layer on the structural and magnetic properties of the heterostructures are still unresolved.

\section{ACKNOWLEDGEMENTS}

The work at Lawrence Berkeley Laboratory is supported by the Director, Office of Energy Research, Office of Basic Energy Sciences, U.S. Department of Energy under contract No. DE-AC03-76SF00098. The work at the University of Maryland is supported by the Office of Naval Research through contract No. N000149510547.

\section{REFERENCES}

1. M.A.Gilleo, "Ferromagnetic Insulators", in Ferromagnetic Materials, Vol.2, Ed. by E.P.Wohlfarth, North-Holland Publishers, New York, 1986; P.I.Slick, "Ferrites for non-microwave applications", ibid; J.Nicolas, "Microwave Ferrites", ibid; J.Smit and H.P.J.Wijn, "Ferrites", John Wiley \&Sons New York, 1959.

2. M.H.Kryder, et.al., "Epitaxial Magnetic Oxides", Air Force Office of Scientific Research Final Report No. AFOSR89-0097; The books in ref.1 also describe the LPE technique and the growth of epitaxial garnet films by LPE.

3. F.J.A.M.Greidanus and W.Bas Zeper, in Materials Research Society Bulletin Special Issue on Optical Storage Materials, Ed. R.J.Gambino, 31(1990); H.Kano, K.Shono, S.Kuroda, N.Kano, N.Koshino and S.Ogawa, IEEE Trans. on Magnetics, 25, 3737(1989);

4. C.F.Buhrer, Jl. of Appl. Phys., 40, 4500(1969); P.Hansen, et.al., Phys. Rev. B27, 6608(1983); K.H.J.Boschow, Jl. of Less Common Metals, 155, 307(1989).

5. M.Gomi and M.Abe, in Materials Research Society Proc. Vol. 150, p121(1989); K.Shono, et.al., ibid; T.Tsuchiya et.al., J1. of Non-Crystalline Solids, 147\&148, 463(1992); T.Hirano, et.al., IEEE Trans. on Magnetics, 28, 3237(1992).

6. "High Density Digital Recording", Eds. K.H.J. Buschow, G.J.Long and F.Gradjean, NATO ASI Series E, Applied Sciences, Vol. 229, Kluwer Academic Publishers, Boston, 1992.

7. W.Reim and J.Schoenes, in "Ferromagnetic Materials", Vol. 5, Ch.2, Eds. K.H.J.Buschow and E.P.Wohlfarth, p133, North-Holland Publishers, Amsterdam, 1990.

8. L.M.Falicov, et.al., "Surface, interface and thin-film magnetism", in Materials Reports section of Jl. of Materials Research, Vol.5, 1299(1990).

9. Papers in Proc. of Materials Research Society Symposium "Magnetic Surfaces, Thin Films and Multilayers", Vol. 231, Eds. S.S.P.Parkin, H.Hopster, J.P.Renard, T.Shinjo and W.Zinn, Materials Research Society, Pittsburgh, PA, 1992.

10. B.M.Simion, G.Thomas, R.Ramesh, V.G.Keramidas and R.L.Pfeffer, Appl. Phys. Lett., 66, 830(1995).

11. B.M.Simion, G.Thomas, R.Ramesh, V.G.Keramidas, E.Marinero and R.L.Pfeffer, J1. of Appl. Phys. , 76,6287(1994). 